\title{
Entity Risk Mechanics
}

\author{
Milík Tichý \\ Faculty of Civil Engineering, Czech Technical University \\ Solinova 7, 16629 Praha 6, Czech Republic \\ milik.tichy@volny.cz \\ www.fsv.cvut.cz \\ Received 1 March 2011; accepted 7 May 2012
}

Abstract

\begin{abstract}
The actual theory of risk is based on phenomenological approach. However, it seems feasible to start thinking about Entities exposed to hazards in a similar way as about structural systems exposed to loads. Concepts of structural mechanics and structural reliability, common in design of constructed facilities, can be implemented into the risk theory, which, in this way, might be upgraded. As a result, new tools could be given to the project risk management enabling better risk predictions than available today. The aspects of the problem are demonstrated by comparing two specific Entities - a Building Structure exposed to loads, and a Company exposed to hazards. Important conceptual analogies between the two Entities are identified.
\end{abstract}

Keywords: Risk theory; structural design; entity; structure; company; risk mechanics

\section{Concepts and the Reference Bases}

The principles of the actual risk theory, as formulated by Kaplan and Garrick [1], are based

- on identifications of hazards, $\mathrm{Hz}$, and hazard scenarios, Sc, and

- on De Moivre's concept of risk (1711, in De Mensura Sortis, see Bernstein, [2]), formulated, in today words, as the product of the hazard scenario probability, $\operatorname{Pr}(\mathrm{Sc})$, and the respective damage, $\mathrm{Dm}(\mathrm{Sc})$.

The identification of hazards and hazard scenarios is based on experience and judgment while the probabilities and damage are established from a priori observations of past events or from a posteriori forecasting of the future. Experience is obviously the governing factor in risk analysis. Thus, it can be stated that the actual risk theory is essentially phenomenological.

A phenomenological approach is typical for the starting periods of whatever science branch. Sooner or later, it will have been followed by elaborate theories based on generalizing mathematical models. Doubtlessly, the science of risk can also be expected to pursue a similar track. A wider, more extensive and more comprehensive theoretical framework for solving systems "hazardsrisks-risk exposed Entity" than that being now dealt with can be foreseen.

The concept of Entity used in the Author's following propositions can be defined as:

Any concrete or abstract thing of interest, including associations among things; for example, a person, object, event, or process that is of interest in the context under consideration, and about which data may be stored in a database. ${ }^{1}$

Thus, "Entity" covers universally all objects and processes of any kind - material, non-material, or mixed.-A few examples of Entities: a manufacturing company, a vacation travel, academic staff of a university, a political party, a philosophical system.

The risk analysis terms, in particular hazard, $\mathrm{Hz}$, hazard scenario, Sc, and risk, Rs, will be applied as outlined by Kaplan and Garrick [1]-Other concepts

${ }^{1}$ See definitions through google.com 
and terms have been recently discussed - see Merkelsen [3].

Time and space shall be specified in any solutions, and so all variables, conditions, and concepts in general, mentioned in the following, shall be always referred to time and space, or possibly also to other reference bases.

Two parameters of time are of interest in analyses of whatever systems: a reference period, $T_{\text {ref, }}$ and its timeline position, $t_{0}$. Similarly as time, the space considered shall be specified in terms of a reference

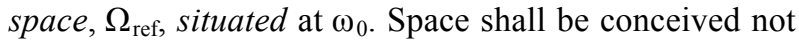
only geometrically. In the risk analyses space may have various meanings: geometrical space, management space, marketing space, operation space, and others.

Both reference bases can be continuous or discrete: $T_{\text {ref }}$ can consist of several time periods (e.g., winter periods, flight time between two airports), $\Omega$ ref can be considered as a set of partial spaces (e.g., classrooms of a school building, departments of a company).

Any of the reference parameters, $T_{\text {ref }}$ and $t_{0}, \Omega_{\text {ref }}$ and $\omega_{0}$, though usually assumed by fixed deterministic values, can be time-dependent, and space-dependent as well. Moreover, they can be random. However, the features of discontinuity, hyperdependences, and randomness of the reference parameters will not be discussed here.

\section{Structure and Company}

For sake of simplicity, only two Model Entities - of substantially different and not related kind - will be compared to show the Author's conception.

Constructed facilities, C.F., i.e. buildings, bridges, dams, and many other engineering works, are exposed to environmental effects that can be of various natures: physical, chemical, economic, political, and many others. C.F.'s are expected to deal with such effects adequately. This means, in technical words, that the respective C.F. will remain fixed in its space and time, and will be sufficiently firm to meet the requirements imposed by its users ${ }^{2}$. These properties are provided by

${ }^{2}$ Other engineering facilities exist whose main role is to move - the Mechanical Facilities (cars, ships, DVD drive mechanics, etc.) -, to transfer energy and information, etc. the carrying structures - foundations, columns, beams, slabs, roof trusses, etc. - joined in one or more systems. Structure will be taken as one of the two Model Entities in this study.

Basic features of structures exposed to loads will be compared here with basic features of another Model Entity: Company, exposed to hazards. A company is described in predominantly management terms, though some amount of physical description cannot be escaped.

Companies and structures are systems that substantially differ by their inherent natures; yet, both have surprisingly much in common. Although the two Model Entities are unrelated, the following comparisons will show striking analogies between both. The left-hand column of the following broken table is reserved for engineering concepts concerning structures, while on the right-hand side risk-related concepts referred to companies are outlined.

\section{Loads and Hazards}

Observe various parallel aspects of loads and hazards:

\begin{tabular}{l|l}
\multicolumn{2}{c}{ Passive role aspect } \\
$\begin{array}{l}\text { Structures are exposed to } \\
\text { loads - Fig. } 1\end{array}$ & $\begin{array}{l}\text { Companies are exposed } \\
\text { to hazards }- \text { Fig. } 2 .\end{array}$
\end{tabular}

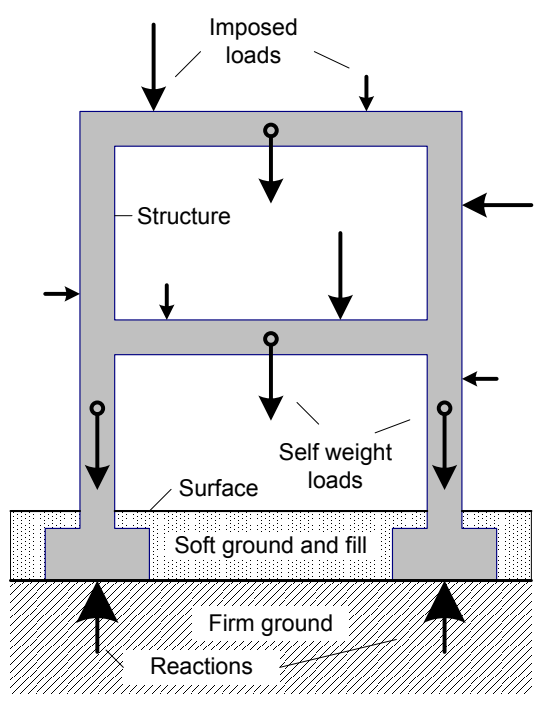

Fig. 1. A simple structure exposed to imposed and self-weight loads. Beams, columns, and footings transmit the load-effects into the firm ground, and the structure is loaded by upside reactions. All loads acting on the structure must be in equilibrium. 


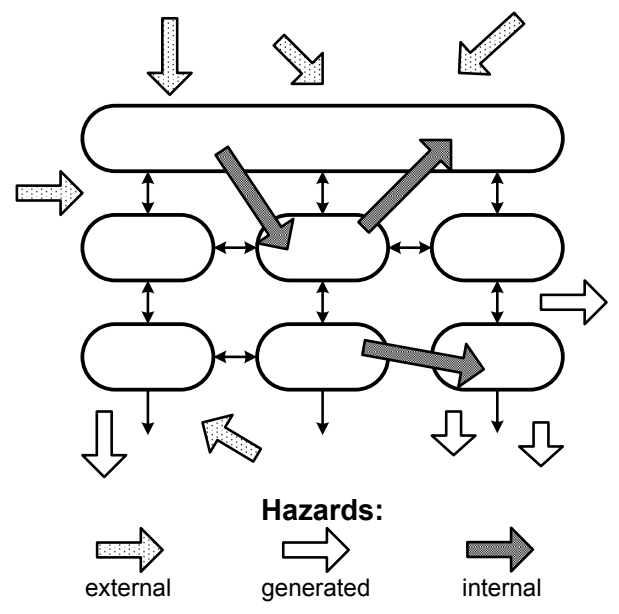

Fig. 2. The organization chart of a company exposed to external and internal hazards and generating hazards to its environment.

\section{Active role aspect}

\begin{tabular}{l|l}
$\begin{array}{l}\text { Structures generate } \\
\text { loads affecting other }\end{array}$ & $\begin{array}{l}\text { Companies generate } \\
\text { hazards to other }\end{array}$ \\
structures and ground. & $\begin{array}{l}\text { companies or to other } \\
\text { Entities (e.g., to the } \\
\text { Environment). }\end{array}$
\end{tabular}

\section{Time aspects}

Loads on structures are usually classified into two principal groups:

- permanent loads,

- transient loads.

\section{Hazards threatening} companies can be classified in the same way:

- permanent hazards,

- transient hazards.

\section{Utility aspects}

- self-weight loads (no terrestrial structure can exist without self-weight),

- imposed loads

(live load, dead load, transient load, wind load, snow load, etc.)

\section{- existence-based} hazards (company cannot avoid them, whether they were active or not),

hazards (affecting the company or created by the company as a result of its activity).
- activity-based

Further aspects, divisions and subdivisions of loads and of hazards, might be easily demonstrated, and the respective classification would become more detailed.

\section{Load Combinations and Hazard Combinations}

In current structures loads never occur in isolated form. As soon as two or more loads occur simultaneously, we talk about a load combination.

As a rule, several load combinations have to be investigated in structural analysis and design

The effect of a single load can be either amplified in combination with other loads, or, on the contrary, diminished.

\section{Load-Effects and Hazard Scenarios}

Structures react to loads in their appropriate way. The resulting load-effects depend upon geometric and physical properties of the carrying system.

Under various arrangements and levels of load the relative distributions of loadeffects are, in general, not identical since they depend upon the response of the structure.
A company is exposed to a set of hazards, many of them can occur simultaneously, and hazard combinations should be faced.

Again, amplification or lessening of the effects of a particular hazard is possible in a hazard combination.

The hazard combination feature is typical in the insurance theory and practice where combined hazards and the ensuing risks are dealt with under different schemes.

One or more hazard scenarios can be identified for each single hazard or for a hazard combination. A set of hazard scenarios must be considered when analyzing a specific hazard affecting a company.

The more complex the company, the more scenarios are possible for any hazard, some of them dominating the others.

Hazard scenarios are the possible results of hazards imposed or proper, and of the company's hazard response (or risk response). 


\section{Structure and Company Properties}

Structures are made of specific materials, which possess sets of properties (strength, ductility, durability, etc.).

The result of these material properties combined with the arrangement of a given structure in a system consisting of carrying members (beams, columns, slabs, arches, etc.) provides for structure's resistance with respect to loads and stiffness under their action.
Companies are built up of units according to various patterns, each unit (e.g., accounting department, shipping and delivering, CEO's Office) having a defined specific role in the respective company's system.

Every unit thus plays a role in company's resistance and stiffness with respect to hazards.

Of course, structures can be conceived as riskologic Entities exposed to hazards, too. Some of structureaffecting hazards stem straight from physical loads, yet there are many hazards that have nothing common with the load category (e.g., hazards due to bad maintenance). On the other hand, a company can be subjected to "loads" that cannot be considered hazards e.g., debt loads, contractual loads.

\section{Kinematic Compatibility Equations And Constitutive Laws}

\subsection{Structure}

How and why structures remain fixed and firm? This crucial double-question had not been asked in the beginnings of the mankind's construction activities; everybody had been happy that buildings, bridges, and other facilities did not systematically break down or fall apart. If, nevertheless, something did happen, the immediate observations were transferred into current practice as experience without going into the background of the event. No theories did exist to generalize the phenomena. Yet, questions started to be raised as soon as extrapolations of experience became dangerous. Answers had been found in due course in a couple of centuries, and, as a result, Structural
Mechanics based on physical laws has developed into a complex system of knowledge.

Stresses in a fixed and firm structural system must be balanced, and all strains and displacements must be mutually compatible. This condition can be symbolically expressed by a set of kinematic compatibility requirements:

$$
\forall t \in T_{\text {ref }}, \forall \omega \in \Omega_{\text {ref }}: \quad f(\sigma, \varepsilon)=0
$$

where $\sigma$ represents the stress state, and $\boldsymbol{\varepsilon}$ the strain and displacement state. Note the time and space quantifiers.

Whenever the equilibrium of forces is lost due to loss of support and compatibility, the structure or its parts start moving until a new equilibrium has been reached. When the ultimate equilibrium state is exceeded, the system collapses and, as a result, the structure ceases to exist as such.

Structural Mechanics does not present the kinematic compatibility requirement in the above symbolic form. As a rule, a system of equations has to be formulated where physical dependences between strains on one side and stresses on the other must be known or assumed. These dependences are usually denoted as constitutive laws of materials (also called constitutive equations).

There always is a relationship between stress and strain in a material. It can be defined mathematically in terms of a function $g(\sigma, \varepsilon)$ based on experimental observations, the graph of which is often called the "stress-strain diagram", SSD. This function can have various forms in dependence upon the properties of the material applied, loading rate, temperature, and many other factors. For illustration, two simple SSD's, typical for many materials, are shown in Fig. 3 . $^{3}$

\subsection{Company}

An analogous set of concepts can be followed considering the company model. However, a specific concept shall be introduced, viz. the concept of concern, $\mathrm{Cn}$.

Risk generates concern in individuals and groups at various time and space positions (managers, family heads, farmers, sailors, parliaments, nations, etc.).

\footnotetext{
${ }^{3}$ For certain materials under specific conditions SSD can be assumed linear, and is well known from elementary physics as Hooke's Law.
} 
Concern can be measured by methods of psychology, and dependence between risk and concern can be empirically found for each case. Such dependence can be taken as the constitutive law of the company. It can be graphically presented as a risk-concern diagram, RCD, Fig. 4.
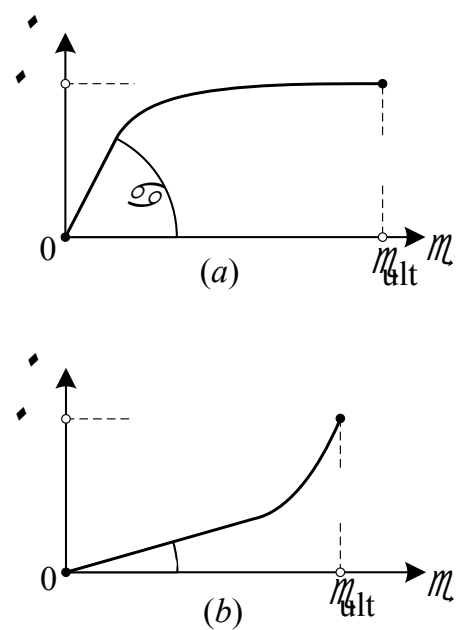

Fig. 3. Stress-strain diagram, SSD, of a structural material: (a) - material softens with growing strain, (b) - material hardens with growing strain.
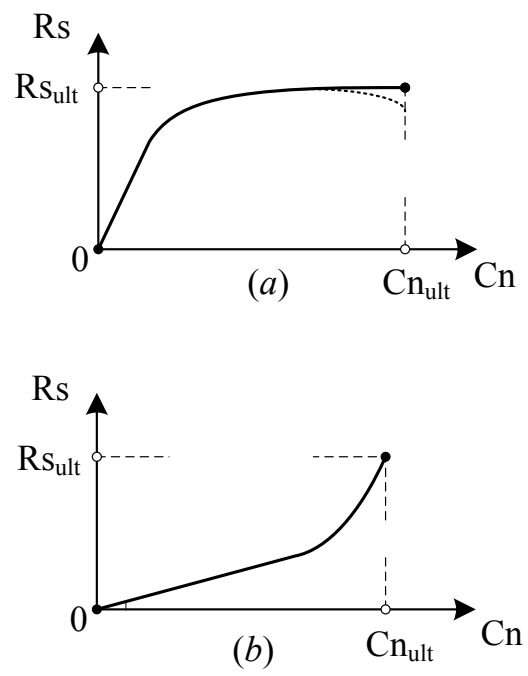

Fig. 4. Risk-concern diagram, RCD, of a company: $(a)-$ risk-averse company, $(b)$ - risk-reverse company.

It can be approximately said that concern, Cn, covers the qualitative aspect of a hazard situation while risk, Rs, expresses the quantitative aspect. Again, RCD can be mathematically described by a function, $g(\mathrm{Rs}, \mathrm{Cn})$, which seems to be a challenging task for future investigations.
Now, a general risk compatibility requirement can be formulated:

$$
\forall t \in T_{\text {ref }}, \forall \omega \in \Omega_{\text {ref }}: f(\mathrm{Rs}, \mathrm{Cn})=0
$$

All company risks, i.e., downside risks and upside risks as well, should enter the requirement. In fact, an equilibrium and compatibility of risks is a condition sine qua non for the survival of any Entity.

Observe that company A with RCD according to Fig. 4a is more risk-sensitive than the company $\mathbf{B}$ with RCD of the type in Fig. 4b. While the company A gets increasingly concerned with small increments of risk, the company $\mathbf{B}$ does not mind, and its concern growth slows down. It is evident that the respective constitutive law depends on the company and its Environment in general concept.

Note that similar considerations can be made for the relationship between hazard severity, Sv, and concern, Cn. Many situations exist where the hazard severity, not the risk, does govern decisions. This particularly happens when important hazards with very small probability of getting materialized are dealt with, and the Precautionary Principle thinking becomes a necessity.

\section{Limit States}

When the intensity of loads increases, or the structural resistance and stiffness diminish ${ }^{4}$, an ultimate equilibrium is reached beyond which any change in load parameters results in collapse. The ultimate equilibrium state is one of several limit states that are a basic concept in the design of structures. Since Mayer [4], 1926, the theory of limit states had successively developed into a now commonly stabilized system, defined, e.g., in structural design codes. Again, analogies between the two Model Entities examined can be observed:

Two basic families of structural limit states are distinguished in the design of structures:

- serviceability limit states, referred to the reliability of structures under "average conditions";
A company's behavior under current conditions is definitely different from its behavior in an exceptional situation. Some functions get intentionally or automatically suppressed, some must be started to

\footnotetext{
${ }^{4}$ The two processes can be mutually dependent.
} 
this group mainly

concerns various forms

of displacements and

deformations of

structural systems,

- ultimate limit states,

dealing with structures

under extreme loads.

\section{Structural Reliability and Risk Acceptability}

The loads and load-effects, and the material properties as well, vary in certain ranges. In general, these variations are random in time and space. Therefore, a simple deterministic request for a fixed and firm structure does not suffice in the design and assessment of C.F. What we are interested in, is how far from the total or partial failure the given structure under the expected load system is. In other words, we want to know whether the structure will be and will remain reliable, i.e. safe, durable, etc., during its assumed use in the time and space envisaged. In practice, we want to design the structure in such a way that its system remains fixed and firm with a certain margin of reliability, $Z(t, \omega)$, during the reference period, $T_{\text {ref, }}$, and in the reference space, $\Omega$ ref, specified. To meet this, a probability-based structural reliability requirement, PSRS, shall be fulfilled (see, e.g., Tichý [5]):

$\forall t \in T_{\text {ref }}, \forall \omega \in \Omega_{\text {ref }}$ :

$$
\operatorname{Pr}(Z(t, \omega) \leq 0) \leq P_{f d}\left(T_{\text {ref }}, \Omega_{\text {ref }}\right)
$$

where $P_{f d}$ is the design value of the failure probability, which is a decision-based parameter, often depending on the reference period and reference space. The magnitude of $P_{f d}$ is established on a set of miscellaneous considerations - economic, psychologic, etc. - of deterministic and statistical character.-For simplicity, the time and space arguments, $(t, \omega)$, are not indicated in the equations that follow.

The margin of safety, $Z$, can be expressed in various ways, in dependence upon the situation considered. In general, $Z$ is formed by a set of elementary random variables describing loads, material properties, geometry of the structure, and also the respective environmental properties the C.F. is exposed to. Thus $Z$ is a time-and-space-dependent random variable.-In simplification, $Z$ can be understood as the highest load the respective structure is able to carry.
If now the analogy track is followed, a probabilitybased risk acceptability requirement, PRAR, can be formulated for a company:

$\forall t \in T_{\text {ref }}, \forall \omega \in \Omega_{\text {ref }}: \operatorname{Pr}\left(\mathrm{Rs}_{\mathrm{acc}} \leq 0\right) \leq P_{\text {adm }}\left(T_{\text {ref }}, \Omega_{\text {ref }}\right)$

where $\mathrm{Rs}_{\mathrm{acc}}$ is the acceptable risk, and $P_{\mathrm{adm}}$ is an admissible probability, the latter being dependent on $T_{\text {ref }}$ and $\Omega_{\text {ref. }}$.

The acceptable risk is defined by (cf. Fig. 5)

$\mathrm{Rs}_{\mathrm{acc}}=\mathrm{Rs}_{\mathrm{cap}}-\mathrm{Rs}_{\mathrm{exp}}$

where $\mathrm{Rs}_{\text {cap }}$ is the risk capacity, i.e., the risk the company is able to carry at ultimate conditions, and $\mathrm{Rs}_{\text {exp }}$ is the expected risk the company will supposedly be exposed to from external and internal hazards. The expected risk shall be established by analyzing the company's risk portfolio, and the risk capacity depends upon company's properties and its environment in broad sense.

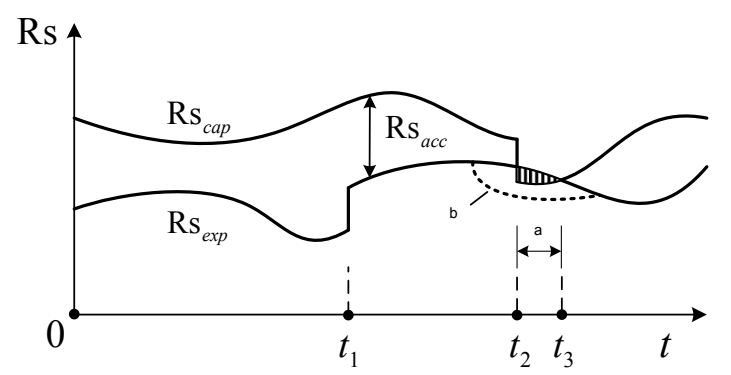

Fig. 5. Risk capacity, $\mathrm{Rs}_{\text {cap }}$, and expected risk, $\mathrm{Rs}_{\text {exp }}$, are mutually independent random processes. Both can be continuous in time with occasional random or non-random discontinuities at $t_{1}, t_{2}$. At $t_{2}$ the acceptable risk, $\mathrm{Rs}_{a c c}$, becomes negative for a certain period, a. In case the risk capacity process is not governable by the company's management, a temporary solution, $\mathbf{b}$, must be found in reducing the expected risk, $\mathrm{Rs}_{\exp }$.- Similar situations can be demonstrated for a structure when it gets temporarily overloaded at some period of its lifetime.

Note that, for simplicity, PRAR is presented here in scalar form while it definitely is a multidimensional requirement.

Both the expected risk and the risk capacity are timeand-space dependent and random, and so is the acceptable risk. For illustration, Fig. 5 shows the time dependence of the expected risk, $\mathrm{Rs}_{\mathrm{exp}}$, and of the risk capacity, $\mathrm{Rs}_{\text {cap }}$. Observe that the curves representing the two quantities may not be continuous. At $t_{1}$ there is a jump rise of the expected risk, and at $t_{2}$ the risk capacity suddenly drops; the jumps can be caused, e.g., by a change in regulations that govern the company's activity and the company's environment. Measures must be 
taken to cope with such situations and the expected risk must be treated to pass periods of a possible company failure.

The admissible probability, $P_{\text {adm }}$, refers to the limit state when the company becomes a hazard to itself and to its environment. While the concept of $P_{f d}$ in PSRS is more or less well understood in the structural engineering community, not too much can be said about $P_{\text {adm }}$ at this moment. The risk resistance of companies in many countries is often regulated by governments or by international bodies, which are, of course, risk-exposed Entities themselves. E.g., in the banking area, Basel III is a well known regulatory document, similarly as Solvency II is for the insurance industry (see [6], [7], and [8]). If the Structure-Company analogies would be developed, $P_{\text {adm }}$ may become a basic regulatory parameter.

\section{Conclusions}

(1) A principal analogy between Structure and Company can be identified: loads on structures and hazards affecting companies can be considered parallel concepts.

(2) The ensuing analogies between concepts of Structural Mechanics and concepts of Risk Analysis can be concised into the following list:

\begin{tabular}{c|c}
$\begin{array}{c}\text { Structural } \\
\text { Mechanics }\end{array}$ & $\begin{array}{c}\text { Risk } \\
\text { Analysis }\end{array}$ \\
\hline load & hazard \\
load effect & hazard scenario \\
stress & risk \\
strain & concern \\
reliability & risk acceptability \\
requirement & requirement \\
stress-strain & risk-concern \\
diagram & diagram
\end{tabular}

(3) The analogy approach can be applied in comparing Structures with other Entities of very different kind.

(4) Principles of Structural Mechanics and Structural Reliability Theory can be applied in risk analyses of financial institutions of different kind, political bodies, biological systems, and scores of many other material and non-material Entities of surprisingly differing kind.
(5) Many research topics are offered by the analogy approach. Theoretical as well as experimental research will be needed to define mathematical models of Entity risk behavior.

(6) There is a good chance that an Entity Risk Mechanics be built, analogous to the existing Structural Mechanics.

(7) The actual risk theory can be raised from its phenomenological platform to a higher level.

Note that in the foregoing discussion the term "Company" can be simply substituted by "Entity".

\section{Acknowledgements}

I would like to thank Professor Jiří Šejnoha for his insightful suggestions concerning some aspects of the analogies presented. However, any errors are my responsibility.

\section{References}

[1] S. Kaplan, B. J. Garrick, On the quantitative definition of risk, Risk Analysis, 1(1) (1981), 11-27

[2] P. L. Bernstein, Against the Gods (John Wiley, New York, 1996)

[3] H. Merkelsen, The constitutive element of probabilistic agency in risk: a semantic analysis of risk, danger, chance, and hazard, Journal of Risk Research, 14(7) (2011), 881-897

[4] M. Mayer, Sicherheit der Bauwerke und ihre Berechnung nach Grenzkräften anstatt nach zulässigen Spannungen (Julius Springer, Berlin, 1926)

[5] M. Tichý, Applied Methods of Structural Reliability (Kluwer Academic Publishers, Dordrecht, 1994)

[6] A global regulatory framework for more resilient banks and banking systems - Basel III, (Basel, Bank for International Settlements, 2010, rev. 2011)

[7] International framework for liquidity risk measurement, standards and monitoring - Basel III (Basel, Bank for International Settlements, 2010)

[8] Directive 2009/138/EC of the European Parliament and of the Council of 25 November 2009 on the taking-up and pursuit of the business of Insurance and Reinsurance - Solvency II 